\title{
Inactivating mutations of acetyltransferase genes in B-cell lymphoma
}

Laura Pasqualucci ${ }^{1,2}$, David Dominguez-Sola ${ }^{1}$, Annalisa Chiarenza ${ }^{1}$, Giulia Fabbri ${ }^{1}$, Adina Grunn ${ }^{1}$, Vladimir Trifonov ${ }^{3}$, Lawryn H. Kasper ${ }^{4}$, Stephanie Lerach ${ }^{4}$, Hongyan Tang ${ }^{1}$, Jing Ma ${ }^{5}$, Davide Rossi ${ }^{6}$, Amy Chadburn ${ }^{7}$, Vundavalli V. Murty ${ }^{1,2}$, Charles G. Mullighan ${ }^{8}$, Gianluca Gaidano ${ }^{6}$, Raul Rabadan ${ }^{3}$, Paul K. Brindle ${ }^{4}$ \& Riccardo Dalla-Favera ${ }^{1,2,9}$

B-cell non-Hodgkin's lymphoma comprises biologically and clinically distinct diseases the pathogenesis of which is associated with genetic lesions affecting oncogenes and tumour-suppressor genes. We report here that the two most common types-follicular lymphoma and diffuse large B-cell lymphoma-harbour frequent structural alterations inactivating CREBBP and, more rarely, EP300, two highly related histone and non-histone acetyltransferases (HATs) that act as transcriptional co-activators in multiple signalling pathways. Overall, about $39 \%$ of diffuse large B-cell lymphoma and $41 \%$ of follicular lymphoma cases display genomic deletions and/or somatic mutations that remove or inactivate the HAT coding domain of these two genes. These lesions usually affect one allele, suggesting that reduction in HAT dosage is important for lymphomagenesis. We demonstrate specific defects in acetylation-mediated inactivation of the BCL6 oncoprotein and activation of the p53 tumour suppressor. These results identify CREBBP/EP300 mutations as a major pathogenetic mechanism shared by common forms of B-cell non-Hodgkin's lymphoma, with direct implications for the use of drugs targeting acetylation/deacetylation mechanisms.

Diffuse large B-cell lymphoma (DLBCL) represents the most common form of B-cell non-Hodgkin lymphoma (B-NHL), accounting for about $30 \%$ of new diagnoses and also arising as a frequent clinical evolution of follicular lymphoma ${ }^{1}$. The molecular pathogenesis of DLBCL is associated with multiple genetic lesions that segregate in part with individual phenotypic subtypes, namely germinal-centre B-cell-like (GCB) and activated B-cell-like (ABC) DLBCL, suggesting the involvement of distinct oncogenic pathways ${ }^{2-8}$. However, the full spectrum of lesions that contribute to malignant transformation remains unknown. Genome-wide efforts toward the identification and functional characterization of the entire set of structural alterations present in the DLBCL genome are required for a complete understanding of its pathogenesis ${ }^{9}$.

Towards this end, we have integrated next-generation wholeexome sequencing analysis of seven DLBCL cases and genome-wide high-density single nucleotide polymorphism (SNP) array analysis of 72 DLBCL cases. This combined approach led to the identification of over 450 loci that are affected by somatic point mutations and/or by recurrent, focal gene copy number aberrations. Among those that have been independently validated, the most commonly involved regions were those harbouring the acetyltransferase genes $C R E B B P$ $(C B P)$ and EP300 (p300). CREBBP encodes a highly conserved and ubiquitously expressed nuclear phosphoprotein that, together with the closely related protein EP300, belongs to the KAT3 family of histone/protein lysine acetyltransferases ${ }^{10,11}$. CREBBP and EP300 function as transcriptional coactivators for a large number of DNAbinding transcription factors involved in multiple signalling and developmental pathways, by modifying lysine residues on both histone and non-histone nuclear proteins ${ }^{12,13}$.

CREBBP and EP300 enhance transcription by multiple mechanisms, including: (1) targeted acetylation of chromatin ${ }^{12,13}$; (2) acetylation of transcriptional activators (such as the tumour suppressors p53 and GATA 1$)^{14-17}$; and (3) acetylation-mediated inactivation of transcriptional repressors (such as the DLBCL-associated oncogene BCL6) ${ }^{18}$. Additionally, both molecules were found to exhibit ubiquitin ligase activity ${ }^{19,20}$. Consistent with the involvement in critical cellular functions, homozygous null mice for either Crebbp or Ep300 are early embryonic lethal ${ }^{21,22}$, and the same is true for compound Crebbp/ Ep300 double heterozygous mice ${ }^{21,22}$. Haploinsufficiency of CREBBP (and, rarely, EP300) is responsible for Rubinstein-Taybi syndrome, an autosomal congenital disorder characterized by mental and growth retardation, skeletal abnormalities and predisposition to tumour development $\mathrm{t}^{23-25}$. Somatic mutations of these two genes are exceedingly rare in epithelial cancers ${ }^{26-28}$, and only three cases were reported to carry EP300, but not CREBBP mutations in haematologic malignancies ${ }^{29,30}$. Additionally, chromosomal translocations of CREBBP are associated with an infrequent type of acute myeloid leukaemia and with therapy-related acute myeloid leukaemia and myelodysplastic syndrome, although the precise consequences of these lesions have not been fully elucidated ${ }^{31-33}$.

\section{Monoallelic lesions of CREBBP in DLBCL}

Following initial observations from whole-exome sequencing analysis of seven DLBCL cases and paired normal DNAs, we performed targeted

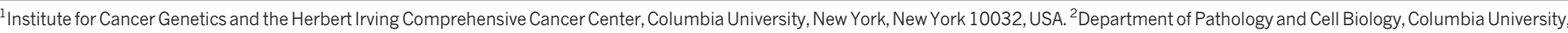

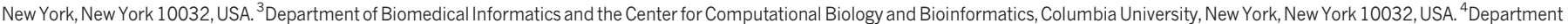

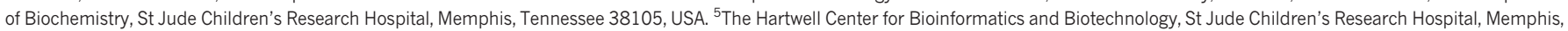

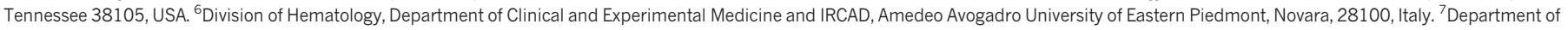
Pathology, Northwestern University, Feinberg School of Medicine, Chicago, Illinois 60611, USA. ${ }^{8}$ Department of Pathology, St Jude Children's Research Hospital, Memphis, Tennessee 38105, USA.

${ }^{9}$ Department of Genetics and Development, Columbia University, New York, New York 10032, USA.
} 

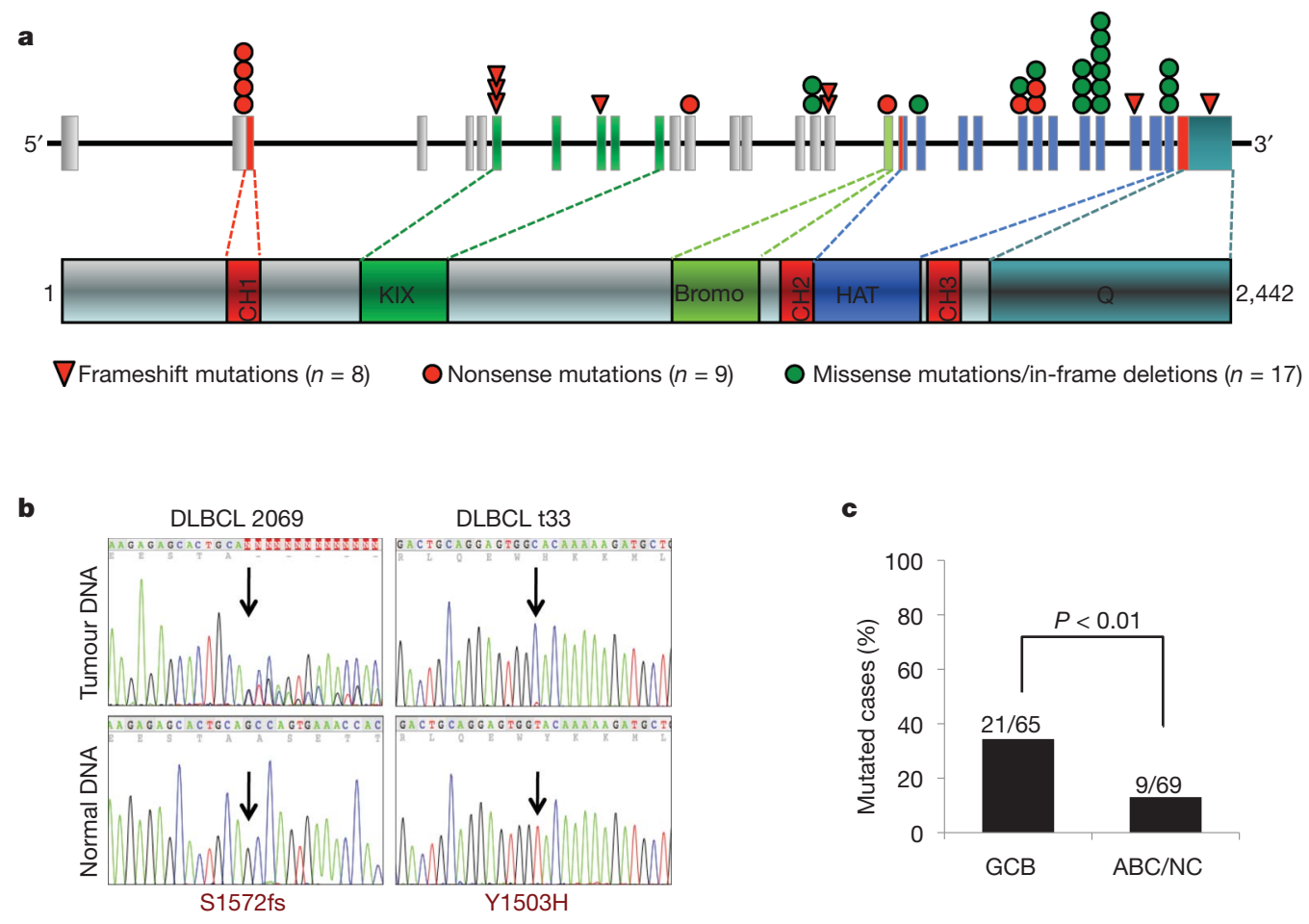

Figure $1 \mid$ The CREBBP gene is mutated in DLBCL. a, Schematic diagram of the $C R E B B P$ gene (top) and protein (bottom). Exons are colour-coded according to the corresponding protein functional domains $(\mathrm{CH}$, cysteinehistidine rich; KIX, CREB-binding; Bromo, bromodomain; HAT, histone acetyltransferase; Q, polyglutamine stretch). Colour-coded symbols depict

re-sequencing of the entire CREBBP coding exons in 134 DLBCL samples representative of major phenotypic subtypes. This analysis revealed a total of 34 sequence variants distributed over 30 samples and the somatic origin of which was documented by analysis of paired normal DNAs (available in eight cases)(Fig. 1a and b, and Supplementary Table 1). Of these variants, 17 (50\%) were inactivating events, including nonsense mutations $(n=9)$, frameshift insertions/deletions $(n=7)$ and mutations at consensus splice donor/splice acceptor sites $(n=1)$, which generate aberrant transcripts carrying premature stop codons. On the basis of their distribution along the CREBBP protein, these mutations are all predicted to cause the elimination or truncation of the HAT domain (Fig. 1a and Supplementary Table 1). The remaining variants included three in-frame deletions and 14 missense mutations, primarily within the HAT domain (Fig. 1a and Supplementary Table 1), suggesting that they may be functionally important (see below). Although CREBBP mutations were identified in both DLBCL phenotypic subtypes, their frequency was significantly higher in GCBDLBCL, where they account for about $32 \%$ of the cases $(n=21 / 65)$ as compared to $13 \%$ in ABC/non-classified (NC) DLBCL $(n=9 / 69$; $P<0.01$ ) (Fig. 1c).

High-density SNP array analysis, available for 72 samples from the same panel, and fluorescence in situ hybridization (FISH) analysis revealed the presence of monoallelic deletions encompassing or internal to the CREBBP locus in eight additional cases (five GCBDLBCL and three ABC/non-GC-DLBCL), and a homozygous deletion in one patient (Fig. 2a and b, and Supplementary Fig. 1). Notably, the loss of genetic material was smaller than 240 kilobases $(\mathrm{kb})$ in four cases and, in two patients (2147 and 2043), involved only a limited subset of CREBBP exons, thereby identifying this gene as the specific target (Fig. 2a and Supplementary Table 2). In two additional samples for which copy number data were not available, direct sequencing analysis revealed a hemizygous missense mutation, reflecting either the loss of the second allele or copy neutral loss of heterozygosity distinct types of mutations. $\mathbf{b}$, Sequencing traces of representative mutated DLBCL tumour samples and paired normal DNA; arrows point to the position of the nucleotide change (amino acid change shown at the bottom). c, Distribution of CREBBP mutations in major DLBCL subtypes; the actual number of mutated samples over the total analysed is given. NC, not classified.

(Supplementary Table 1). When combining the sequencing data with the copy number data, CREBBP mutations and deletions were found to be mutually exclusive in most samples, revealing a predominantly monoallelic distribution ( $n=33 / 39$ cases) (Fig. 2 c). Rare instances of biallelic genetic lesions include the homozygous loss, a missense mutation with loss of heterozygosity (two primary biopsies), biallelic nucleotide substitutions (two cases), and a frameshift deletion with missense mutation of the second allele in the OCI-Ly8 cell line. Thus, $29 \%$ of all DLBCL patients $(n=39 / 134)$, corresponding to $41.5 \%$ of GCB-DLBCL and $17 \%$ of ABC-DLBCL, harbour genomic alterations affecting the $C R E B B P$ gene (Fig. 2d).

\section{Frequent mutations of CREBBP in follicular lymphoma}

We next analysed various types of mature B-NHL, including follicular lymphoma, Burkitt lymphoma, marginal-zone lymphoma and chronic lymphocytic leukaemia. Mutations analogous to those found in DLBCL were frequent in follicular lymphoma (32.6\%, with 16 events distributed over 15/46 cases), but not in other lymphoma types, suggesting a specific role in the pathogenesis of these two diseases (Supplementary Fig. 2). This analysis also revealed the existence of several mutational hotspots at specific codons within the HAT domain, including R1446 (also mutated in B-cell acute lymphoblastic leukaemia $^{34}$ ), Y1503 and D1435; in addition, a 3-base-pair (bp) inframe deletion causing the loss of $\mathrm{S} 1680$ was observed in five cases, suggesting a functional role for this currently uncharacterized serine (Supplementary Table 1). None of these recurrent changes was detected in paired normal DNA, indicating that they do not represent germline polymorphisms. Although copy number data were not available for the same follicular lymphoma panel, array-CGH analysis performed on a distinct data set showed deletions spanning the CREBBP locus in only $1 / 68$ cases (not shown). Collectively, these findings identify somatic mutations of CREBBP as a common event in follicular lymphoma. 


\section{a}

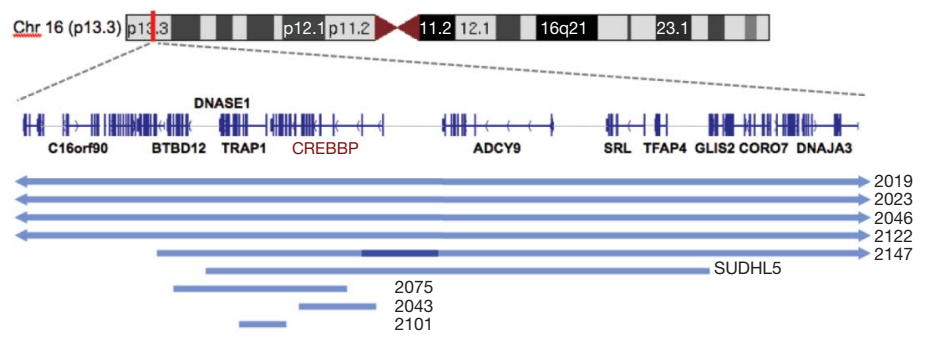

c

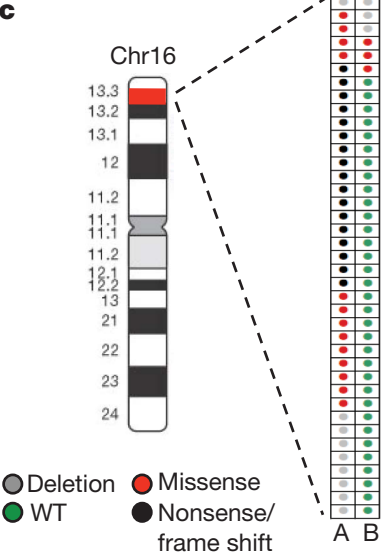

d Nonsense mutation
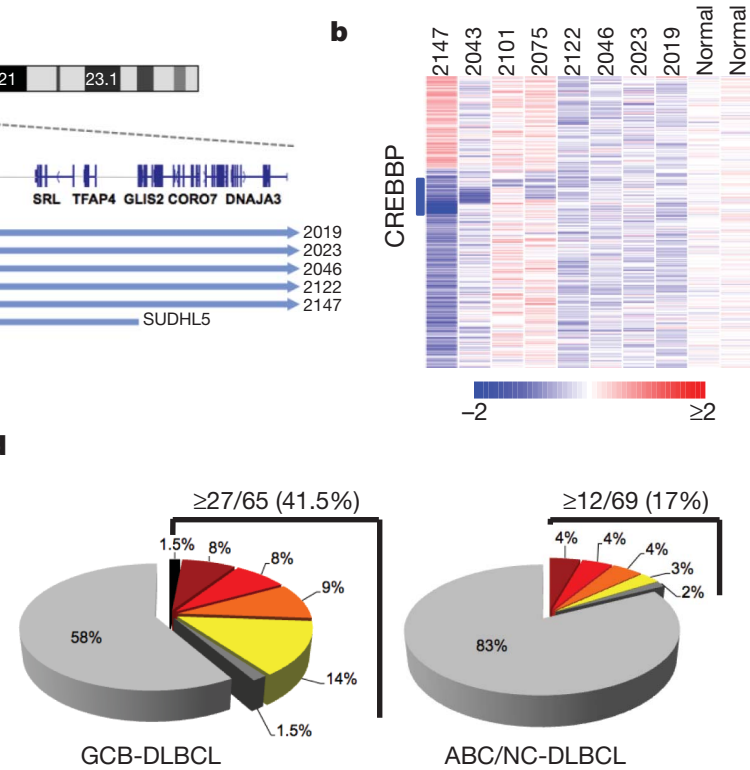

Homozygous deletion $\square$ Missense mutation, HAT domain Heterozygous deletion $\square$ Missense mutation, outside HAT Frameshift mutation $\square$ Unmutated

Figure $2 \mid$ Mutations and deletions of $C R E B B P$ are predominantly monoallelic. a, Map of the genomic region encompassing CREBBP and its neighbouring genes on chromosome 16p13.3. Blue lines below the map indicate the extent of the deletions identified in nine DLBCL samples, with the darker blue segment corresponding to a homozygous loss. b, dChipSNP heatmap showing median-smoothed log2 copy number ratio for eight DLBCL biopsies harbouring $C R E B B P$ deletions, and two normal DNAs. A vertical blue bar

\section{EP300 mutations in DLBCL and follicular lymphoma}

Given the significant structural and functional similarities between $C R E B B P$ and $E P 300$, we investigated whether this second member of the KAT3 acetyltransferase family is also targeted by structural alterations in B-NHL. Mutational analysis of the same panel identified 19 sequence variants leading to amino acid changes $(n=11)$, in-frame deletions $(n=2)$, and premature stop codons due to frameshift deletions, aberrant splicing or nonsense mutations $(n=6)$ (Supplementary Fig. 3a and Supplementary Table 3). These lesions were found in $10 \%$ of DLBCL $(n=13 / 134)$ and $8.7 \%$ of follicular lymphoma samples $(n=4 / 46)$, but were almost absent in other B-NHLs (Supplementary Fig. 3b). Seven additional DLBCLs harboured monoallelic deletions spanning, although not limited to, the EP300 locus (Supplementary Fig. 3c, Supplementary Table 4 and data not shown). Notably, structural alterations of CREBBP and EP300 coexist in only a minority of the affected cases ( $n=6 / 53$ DLBCL and 0/19 follicular lymphoma) (Supplementary Fig. $3 \mathrm{~d}$ ), suggesting that inactivation of these loci is at least in part functionally equivalent. Thus, overall about $39 \%$ of all DLBCL and at least $41 \%$ of follicular lymphoma cases (based on mutations only) display structural alterations of KAT3 family genes.

\section{CREBBP and EP300 protein expression}

To compare the CREBBP and EP300 protein levels in normal and transformed B cells, and to investigate the expression of the retained normal allele in cases carrying monoallelic genomic alterations, we examined the expression pattern of these two proteins in reactive human tonsils and in 78 DLBCL primary cases, of which 49 harboured both genes in wild-type configuration, using immunofluorescence and immunohistochemical analysis. Consistent with their reported ubiquitous expression, CREBBP and EP300 were detectable in naive B cells indicates the location of the CREBBP locus; in the red-to-blue colour scale, white corresponds to a normal (diploid) copy number log-ratio, blue corresponds to deletion and red to gain. c, Allelic (A or B) distribution of $C R E B B P$ genetic lesions in individual DLBCL samples. d, Overall frequency of $C R E B B P$ structural alterations in DLBCL subtypes (mutations and deletions, combined).

within the mantle zone and, at higher levels, in germinal-centre B cells (Fig 3a). Notably, most of the monoallelically deleted DLBCL cases were positive for the two proteins, although at reduced levels, demonstrating that the residual wild-type allele is expressed (see Fig. $3 \mathrm{~b}$ and $\mathrm{c}$ for representative examples). Furthermore, reverse-transcription polymerase chain reaction (RT-PCR) amplification and direct sequencing of the ten CREBBP-mutated cell lines invariably showed the presence of the wild-type allele (Supplementary Fig. 4), while western blot analysis using antibodies directed against the amino-terminal portion of CREBBP revealed the expression of a full-length protein, corresponding to the wild-type allele, in most of the cell lines carrying truncating gene mutations, as well as in the monoallelically deleted SUDHL5 cells (Fig. 3d, top panel). Slightly different findings were observed for EP300, where the presence of truncating mutations or deletions was associated with the absence of protein expression in four affected cell lines (Fig. 3d, second panel from top). With the exception of SUDHL2, both CREBBP and EP300 messenger RNAs were readily detected by northern blot analysis in all lines analysed (Fig 3d, panels 4 and 5 from top).

Interestingly, a few additional lines were found to express very low or undetectable protein levels, despite the presence of mRNA and the absence of structural alterations in the corresponding gene (see CREBBP in BJAB and SUHDL2, or EP300 in SUDHL7) (Fig. 3d). Similarly, 6/78 (8\%) DLBCL biopsies from patients with intact alleles appeared to lack expression of the two proteins, either simultaneously (three cases) or individually (two CREBBP +/EP300 - cases and one CREBBP $-/ \mathrm{EP} 300^{+}$case) (Fig. $3 \mathrm{c}$ and e). Thus, the fraction of DLBCL patients with defective CREBBP and EP300 may be higher than that determined on the basis of genetic lesions alone, suggesting that alternative mechanisms of KAT3 gene family inactivation may have a role in this disease. 

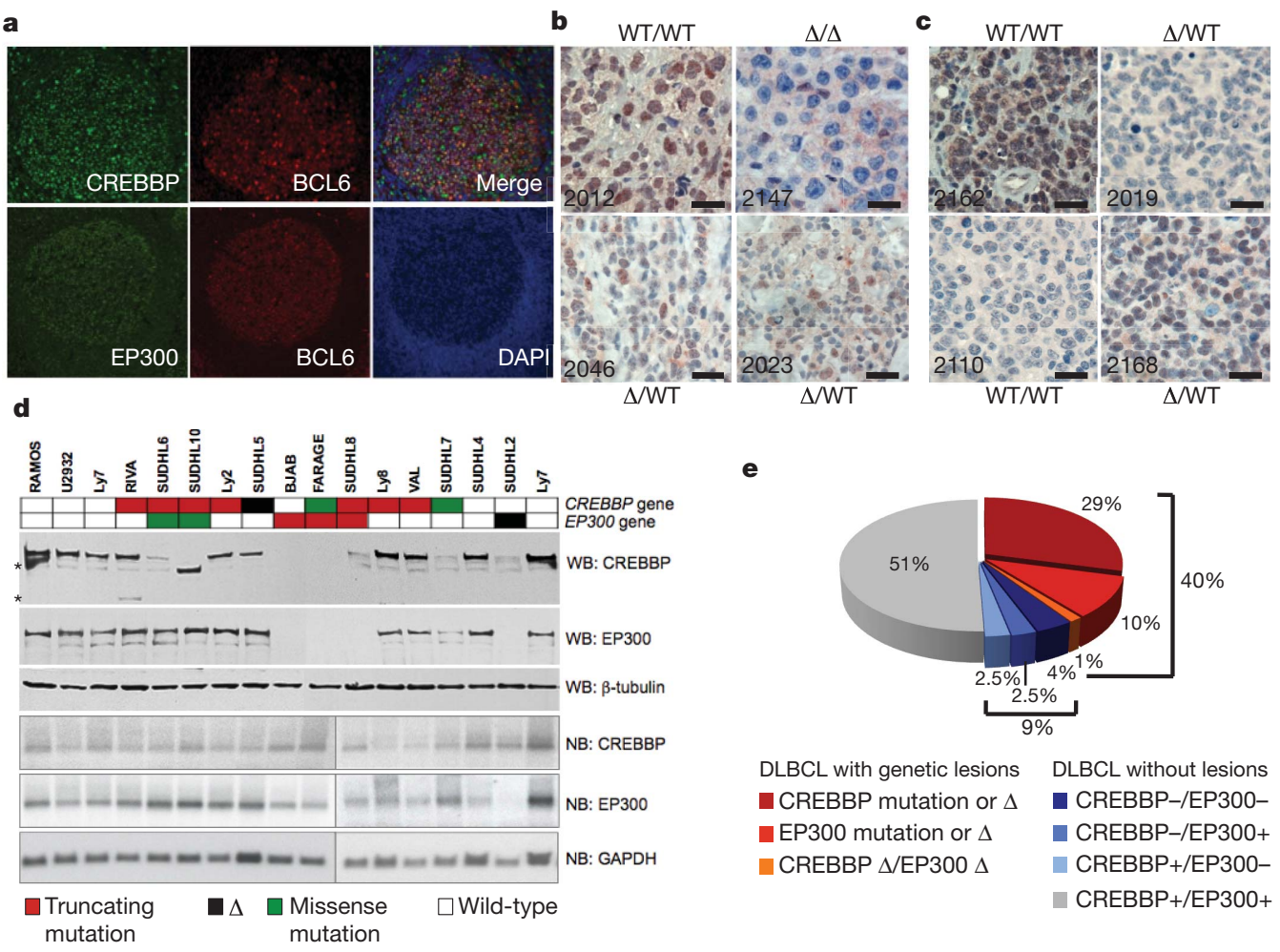

e

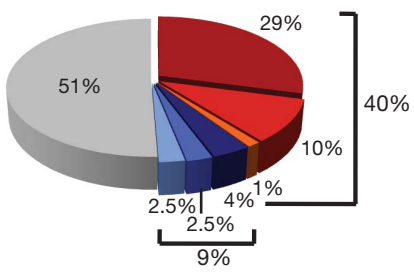

DLBCL with genetic lesions $\quad$ DLBCL without lesions - CREBBP mutation or $\triangle \quad$ CREBBP-/EP300EP300 mutation or $\Delta \quad$ CREBBP-/EP300+ CREBBP $\Delta$ /EP300 $\Delta$ CREBBP+/EP300CREBBP+/EP300+

Figure $3 \mid$ CREBBP and EP300 expression in normal and transformed B-cells. a, Immunofluorescence analysis of reactive tonsils. BCL6 identifies germinal-centre B cells, and DAPI is used to detect nuclei.

b, c, Immunohistochemistry analysis of CREBBP (b) and EP300 (c) protein expression in representative DLBCL biopsies (genomic status as indicated, $\Delta$ is deletion; scale bar, $100 \mu \mathrm{m}$ ). Sample 2147, which harbours a homozygous $C R E B B P$ deletion, serves as negative control. $\mathbf{d}$, Western blot (WB) and

CREBBP missense mutants fail to acetylate BCL6 and p53

The presence of gross gene deletions and the distribution of truncating mutations clearly predict a complete gene inactivation or the loss of multiple key functional domains, including the HAT; on the contrary, the functional consequences of the numerous $C R E B B P$ missense mutations required direct experimental analysis. Notably, of the 30 total events identified, 27 cluster within HAT coding exons, suggesting a selective pressure to alter the CREBBP enzymatic activity (Supplementary Table 1). In particular, 19 mutations were located within a 68amino-acid stretch that is $96 \%$ identical to EP300 and includes the contact surface for coenzyme A (CoA $)^{35}$ (Supplementary Fig. 5). We therefore examined the effect of these mutations on the ability of CREBBP to acetylate known physiologic substrates. We selected BCL6 and p53 because of their biological relevance for germinalcentre development and lymphomagenesis ${ }^{4,36}$. In fact, acetylation of the tumour suppressor p53 is indispensable for its transcriptional activity $^{14,15,37}$, whereas EP300-mediated acetylation of the protooncoprotein BCL6 leads to inactivation of its transcriptional repressor function ${ }^{18}$.

Transient transfection/co-immunoprecipitation assays confirmed that, analogous to EP $300^{18}$, CREBBP binds to and acetylates BCL6, leading to a dose-dependent impairment in its ability to repress a BCL6-responsive reporter gene (Supplementary Fig. 6a and b). We then generated haemagglutinin (HA)-tagged constructs for expression of nine representative CREBBP alleles harbouring missense mutations within $(n=6)$ or immediately outside $(n=2)$ the core HAT domain, as well as a premature stop codon (R1360X) (Fig. 4a). Notably, all of the HAT domain mutant proteins had lost northern blot (NB) analysis of DLBCL cell lines carrying wild-type or aberrant $C R E B B P$ and EP300 alleles (colour-coded as indicated). The aberrant band in SUDHL10 corresponds in size to the predicted $\sim 220$-kilodalton $(\mathrm{kDa})$ CREBBP truncated protein. *Non-specific bands. Tubulin and GAPDH control for total protein and RNA loading, respectively. e, Overall proportion of DLBCL biopsies showing defective CREBBP/EP300 function due to genetic lesions (red scale) and/or lack of protein expression (blue scale).

their ability to acetylate BCL6 (Fig. 4b) and to interfere with its transrepression activity, consistent with the reported role of acetylation in inactivating BCL6 (Fig 4c). Conversely, no significant effects were observed from the C1240R and K1320R proteins or from two additional alleles (P1053L, Q1079H) harbouring mutations outside the HAT domain (Supplementary Table 1 and data not shown), although C1240R appeared to have lost its activity in the reporter assay, suggesting that alternative mechanisms may be involved.

When tested on the tumour suppressor p53, the same core HAT mutants were either impaired or severely attenuated (H1487Y and D1435E) in their acetylation activity, as compared to wild-type CREBBP or to C1240R and K1320R (Fig. 4d). It should be noted that C1240R and K1320R represent a second mutational event in cases carrying an additional truncating CREBBP mutation (see Supplementary Table 1), suggesting the possibility that they represent passenger events or that they confer more subtle functional consequences. Taken together, these findings demonstrate that CREBBP missense mutant alleles have been selected for their defective HAT activity. In particular, the impairment on BCL6 and p53 acetylation is consistent with specific effects of $C R E B B P$ genetic lesions in favouring the constitutive activity of the BCL6 oncogene over the function of the p53 tumour suppressor.

\section{CREBBP mutations reduce affinity for acetyl-CoA binding}

To investigate the mechanisms responsible for the observed loss of function on BCL6 and p53, we examined the effect of CREBBP mutations on subcellular localization, enzyme-substrate complex formation, and enzymatic activity. All mutants tested were correctly localized in 
a

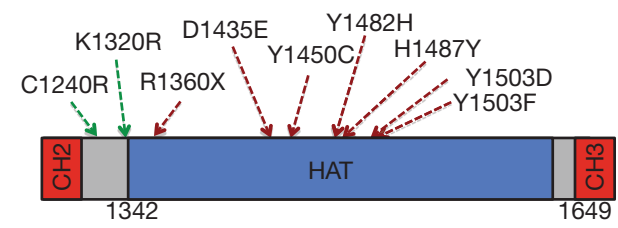

d

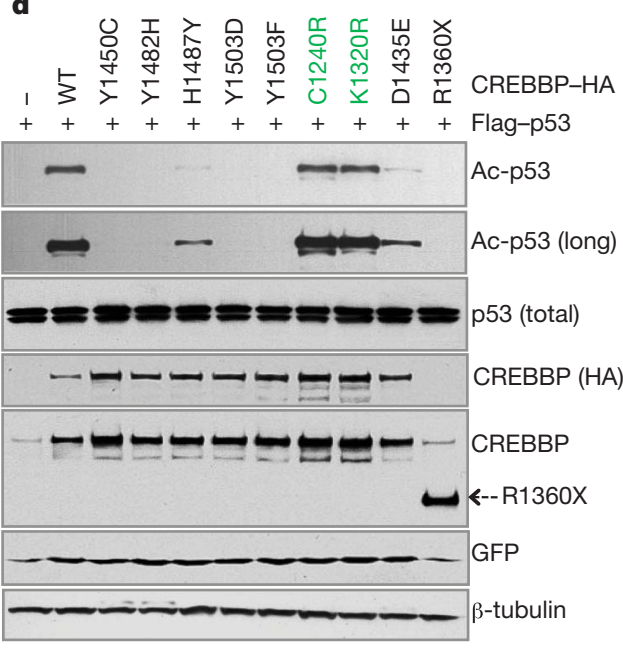

Figure $4 \mid$ CREBBP missense mutations impair its ability to acetylate BCL6 and p53. a, Schematic diagram of the CREBBP HAT and $\mathrm{CH}$ domains, with the CREBBP point mutations tested in $\mathbf{b}-\mathbf{d}$ (in green, residues located immediately outside the HAT domain). $\mathbf{b}$, Acetylation levels of exogenous BCL6 in Flag immunoprecipitates obtained from HEK293T cells co-transfected with wild-type or mutant CREBBP expression vectors. $\beta$-Actin is the input loading control. IP, immunoprecipitation. c, Luciferase reporter assays using a synthetic 5X-BCL6 reporter. The bar graph shows results as relative activity compared to the basal activity of the reporter, set as 1 (mean \pm s.d., $n=2$ ). The

the nuclear compartment and could be efficiently co-immunoprecipitated with BCL6 or p53 (Supplementary Fig. 7 and data not shown), indicating that the inability to acetylate these substrates in vivo was not due to mislocalization or to impaired physical interaction. Instead, in vitro acetylation assays using recombinant glutathione $S$-transferase (GST)-p53 and highly purified, wild-type or mutated CREBBP proteins demonstrated a marked, acetyl-CoA dose-dependent defect in the enzymatic activity of the core HAT mutants (Fig. 5 and Supplementary Fig. 8), suggesting that these changes impair the affinity for acetylCoA (see also Supplementary Fig. 5).

\section{CREBBP mutants are biologically inactive}

To further investigate the consequences of CREBBP missense mutations on its function as a transcriptional co-activator, we examined the response of endogenous $3^{\prime}, 5^{\prime}$-cyclic adenosine monophosphate (cAMP) response element-binding protein (CREB) target genes in mouse embryonic fibroblasts (MEFs) carrying conditional Crebbp and Ep300 double knockout alleles, reconstituted with retroviral vectors expressing either wild-type or DLBCL-associated mutant Crebbp complementary DNAs (Supplementary Fig. 9a), and treated with forskolin and IBMX (two inducers of Protein Kinase A activity). In normal cells, Protein Kinase A-mediated phosphorylation of CREB is required for the recruitment of CREBBP, which in turn leads to transcriptional activation of cAMP-responsive genes ${ }^{38}$. As previously observed $^{39}$, quantitative RT-PCR analysis of CREBBP/EP300-dependent CREB target genes confirmed their upregulation in doubleknockout cells reconstituted with wild-type CREBBP expression constructs (Supplementary Fig. S9b, column 2 from left). In contrast, all four DLBCL-derived CREBBP mutants tested were generally b

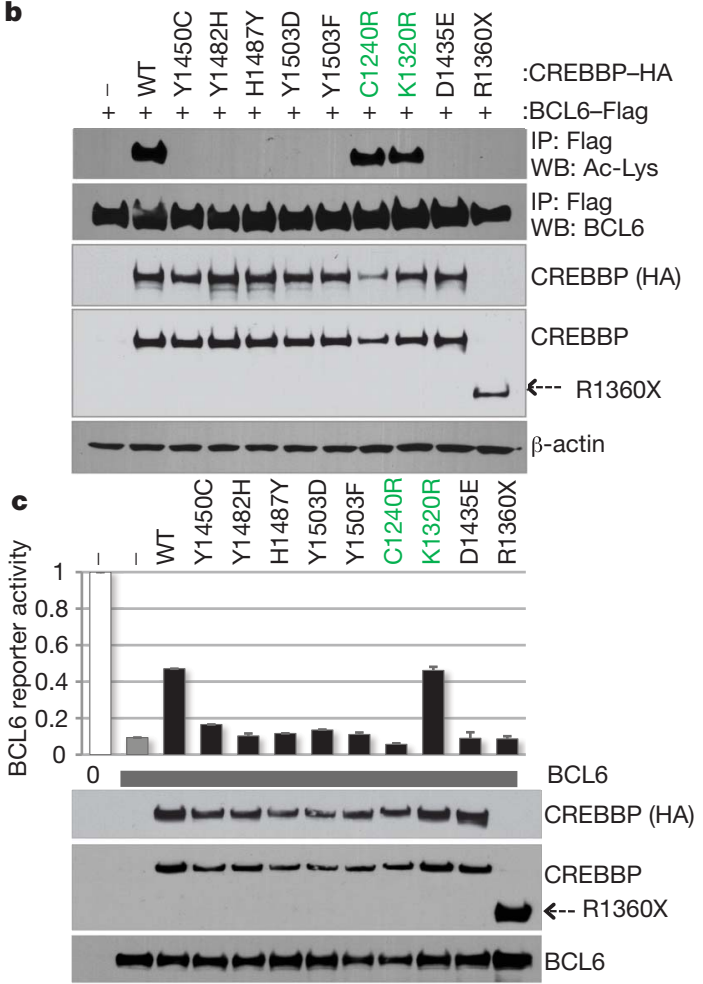

gel shows BCL6 and CREBBP-HA protein levels in the same lysates. Note that the amount of transfected BCL6-encoding and CREBBP-encoding plasmids was adjusted to achieve equal protein amounts. d, p53 acetylation in HEK293T cells co-transfected with the indicated CREBBP expression vectors. The antip53 antibody documents comparable amounts of p53 (exogenous + endogenous). Green fluorescent protein (GFP) monitors for transfection efficiency, and $\beta$-tubulin is used as the loading control. 'Long' indicates long exposure.

deficient for cAMP-responsive transcription, as was the HAT-dead control W1502A/Y1503S ${ }^{40}$ (Supplementary Figs 9b and 10). These mutants were also associated with reduced endogenous histone H3K18 acetylation (Supplementary Fig. 11). Finally, we tested whether the mutant CREBBP polypeptides were proficient in rescuing the proliferative defect of the double-knockout cells, given that this system provides a specific readout for the biological activity of the CREBBP point mutants. Notably, cells transduced with the four DLBCL-associated alleles displayed significantly impaired cell growth compared to wildtype reconstituted cells, as measured in the yellow fluorescent protein (YFP) + (double-knockout) population (Supplementary Fig. 9c). Collectively, the data presented above provide direct experimental evidence for a role of HAT missense mutations in impairing the acetyltransferase activity of CREBBP and its function as a transcriptional coactivator.

\section{Discussion}

These results indicate that inactivation of CREBBP/EP300 represents a common event in the two most frequent forms of B-NHL, namely follicular lymphoma and DLBCL. Previous extensive surveys in malignancies of epithelial origin have reported inactivating mutations of EP300 and CREBBP in exceedingly rare cases ( $<2 \%$ of primary biopsies $)^{26-28}$. Considering their almost total absence in solid tumours, and the finding of recurrent mutations in B-cell acute lymphoblastic leukaemia ${ }^{34}$, our results point to a specific role for CREBBP/EP300 inactivation in the pathogenesis of malignancies derived from B-lymphocytes. Overall, CREBBP/EP300 lesions are among the most frequent structural alterations yet detected in follicular lymphoma and DLBCL, thus representing an important feature of the pathogenesis 
a

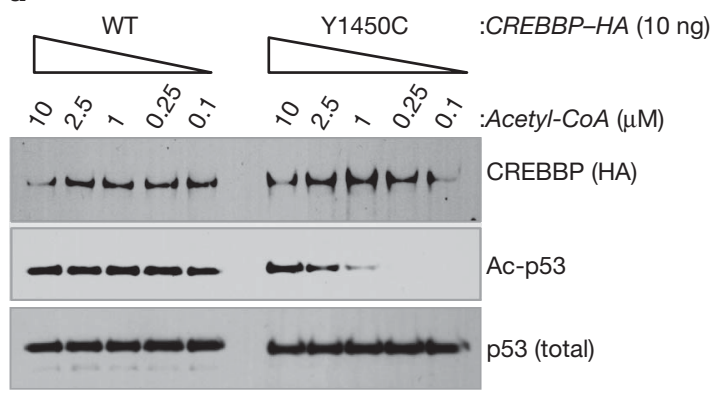

b

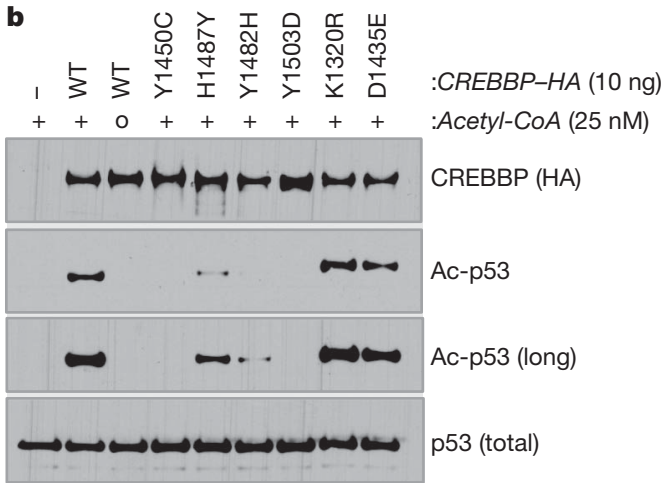

Figure 5 DLBCL-associated mutations in the CREBBP HAT domain decrease its affinity for acetyl-CoA. a, Western blot analysis of in vitro acetyltransferase reactions performed using the WT or mutant Y1450C CREBBP-HA recombinant protein and a GST-p53 substrate in the presence of decreasing amounts of acetyl-CoA. Anti-HA and anti-p53 antibodies document the presence of equivalent amounts of effector and substrate proteins in the reaction. Ac, acetylated. b, In vitro acetyltransferase activity of the indicated CREBBP-HA mutant proteins in the same assay using $25 \mathrm{nM}$ acetyl-CoA.

of these common diseases. Moreover, the observation of significantly reduced CREBBP and EP300 expression levels in a sizeable fraction of DLBCL samples, independent of genetic lesions (Fig. 3e and Supplementary Fig. 12), suggests that additional epigenetic mechanisms may cause reduction of HAT dosage in a larger fraction of tumours.

One key observation of this study is that $C R E B B P / E P 300$ lesions are mostly detected in heterozygosity, suggesting a haploinsufficent role in tumour suppression. This notion is supported by several observations. First, congenital heterozygous mutations of $C R E B B P / E P 300$ are sufficient to cause significant pathologic and developmental phenotypes, including tumorigenesis, in patients with Rubinstein-Taybi syndrome ${ }^{23-25}$, thereby confirming the deleterious effect of reduced HAT activity. Second, a fraction of mice with conditional deletion of Crebbp in mature B cells showed reduced survival past 12 months of age $^{41}$. Haematologic malignancies were also reported in $22 \%$ of constitutive 10-21-month-old Crebbp heterozygous mice and about 30\% of chimeric animals after bone marrow or spleen cell transplantation from Crebbp heterozygotes ${ }^{42}$. Third, in contrast with the abundantly expressed histone deacetylases, HATs are limiting in the cell, suggesting that small dosage variations can have severe biological consequences $^{43}$. Overall, these data provide direct evidence that CREBBP (and, more rarely, EP300) are haploinsufficient tumour suppressors, the specific roles of which in lymphomagenesis will have to be tested by conditional deletion of these alleles in germinal-centre B cells.

Given the global involvement of CREBBP/EP300 on gene transcriptional regulation, it is difficult to predict which cellular targets/ pathways may be critically affected by HAT reduction in lymphomagenesis. At this stage, our results demonstrate that mutant CREBBP and EP300 proteins are deficient in acetylating BCL6 and p53, leading to constitutive activation of the oncoprotein and to decreased p53 tumour suppressor activity. The balance between the activities of these two genes is critical for the regulation of DNA damage responses in mature germinal-centre cells during immunoglobulin gene remodelling ${ }^{36,44}$. Thus, the consequences of BCL6 activity overriding p53 would be an increased tolerance for DNA damage in the context of diminished apoptotic and cell cycle arrest responses.

These results have important therapeutic implications in view of current attempts to use a variety of histone deacetylase inhibitors as anti-cancer drugs. Although the benefits of these compounds have been proved in certain malignancies, such as mature T-cell lymphoproliferative disorders, their efficacy in other common cancers, including B-NHL, is uncertain at this stage $\mathrm{e}^{45}$. The findings of this study suggest that the use of histone deacetylase inhibitors has a rational basis in B-NHL, because it may contribute to re-establishing physiologic acetylation levels. On the other hand, their efficacy should be re-evaluated by stratifying patients based on the presence of HAT defects as well as by testing the numerous histone deacetylase and sirtuin inhibitors with target specificity.

\section{METHODS SUMMARY}

Mutation analysis. The complete coding sequences and exon/intron junctions of CREBBP and EP300 were analysed by PCR amplification and direct sequencing of whole-genome amplified DNA using the oligonucleotides reported in Supplementary Tables 5 and 6 . Mutations were confirmed from both strands on independent PCR products amplified from genomic DNA, and their somatic origin was documented by analysis of matched normal DNA in available cases. SNP array analysis. This was performed using Affymetrix Genome-Wide 6.0 Arrays and a computational workflow, which is described in detail in the Supplementary Information.

In vivo and in vitro characterization of HAT activity. The ability of CREBBP mutants to acetylate BCL6 and p53 was assessed in human embryonic kidney (HEK293T) cells after co-transfection of pCMV-Flag-BCL6 (or pCIN4-Flagp53) with plasmids encoding wild-type versus mutant HA-tagged mouse Crebbp. BCL6 acetylation was evaluated on Flag/M2 immunoprecipitates using antibodies directed against acetyl lysines; for p53, western blot analysis was performed on whole-cell extracts using a specific anti-acetylated p53 antibody. The amounts of exogenous CREBBP were monitored using anti-HA and anti-CREBBP (A22, Santa Cruz Biotechnology) antibodies. In vitro acetylation assays were performed using recombinant GST-p53 and purified CREBBP-HA proteins, in the presence of the indicated amounts of acetyl-CoA.

Transient transfection/reporter gene assays. The effect of CREBBP on BCL6dependent transcription was assessed in HEK293T cells co-transfected with a luciferase reporter vector containing five BCL6 consensus binding sites upstream of the SV40 promoter (5XBCL6) and the pCMV-Flag-BCL6 construct, in the absence or presence of wild-type versus mutant CREBBP-HA expression vectors (see the online-only Methods).

Reconstitution of Crebbp/Ep300 null MEFs. Crebbp $p^{\text {flox } / f l o x} ; E p 300^{\text {flox/flox }} ; \mathrm{YFP}^{+}$ conditional (double knockout) MEFs have been described ${ }^{39}$. CREBBP expression was restored by retroviral infection with constructs encoding for HA-tagged wildtype or mutant Crebbp, and cells were analysed for H3K18 acetylation, cAMPdependent transcriptional responses and cell proliferation (see the online-only Methods).

Full Methods and any associated references are available in the online version of the paper at www.nature.com/nature.

\section{Received 25 June; accepted 2 December 2010.}

1. Swerdlow, S. H. et al. WHO Classification of Tumours of Haematopoietic and Lymphoid Tissues (International Agency for Research on Cancer (IARC), Lyon, 2008).

2. Compagno, M. et al. Mutations of multiple genes cause deregulation of NF- $\mathrm{kB}$ in diffuse large B-cell lymphoma. Nature 459, 717-721 (2009).

3. Davis, R. E. et al. Chronic active B-cell-receptor signalling in diffuse large B-cell lymphoma. Nature 463, 88-92 (2010).

4. Klein, U. \& Dalla-Favera, R. Germinal centres: role in B-cell physiology and malignancy. Nature Rev. Immunol. 8, 22-33 (2008).

5. Lenz, G. et al. Oncogenic CARD11 mutations in human diffuse large B cell lymphoma. Science 319, 1676-1679 (2008).

6. Lenz, G. \& Staudt, L. M. Aggressive lymphomas. N. Engl. J. Med. 362, 1417-1429 (2010).

7. Mandelbaum, J. BLIMP1 is a tumor suppressor gene frequently disrupted in activated B-cell like diffuse large B-cell lymphoma. Cancer Cell 18, 568-579 (2010). 
8. Morin, R. D. et al. Somatic mutations altering EZH2 (Tyr641) in follicular and diffuse large B-cell lymphomas of germinal-center origin. Nature Genet. 42, $181-185$ (2010).

9. Downing, J. R. Cancer genomes-continuing progress. N. Engl. J. Med. 361, 1111-1112 (2009).

10. Goodman, R. H. \& Smolik, S. CBP/p300 in cell growth, transformation, and development. Genes Dev. 14, 1553-1577 (2000).

11. Kalkhoven, E. CBP and p300: HATs for different occasions. Biochem. Pharmacol. 68, 1145-1155 (2004).

12. Bannister, A. J. \& Kouzarides, T. The CBP co-activator is a histone acetyltransferase Nature 384, 641-643 (1996).

13. Ogryzko, V. V., Schiltz, R. L., Russanova, V., Howard, B. H. \& Nakatani, Y. The transcriptional coactivators p300 and CBP are histone acetyltransferases. Cell 87 953-959 (1996).

14. Gu, W., Shi, X. L. \& Roeder, R. G. Synergistic activation of transcription by CBP and p53. Nature 387, 819-823 (1997)

15. Lill, N. L., Grossman, S. R., Ginsberg, D., DeCaprio, J. \& Livingston, D. M. Binding and modulation of p53 by p300/CBP coactivators. Nature 387, 823-827 (1997)

16. Avantaggiati, M. L. et al. Recruitment of p300/CBP in p53-dependent signal pathways. Cell 89, 1175-1184 (1997)

17. Blobel, G. A., Nakajima, T., Eckner, R., Montminy, M. \& Orkin, S. H. CREB-binding protein cooperates with transcription factor GATA-1 and is required for erythroid differentiation. Proc. Natl Acad. Sci. USA 95, 2061-2066 (1998).

18. Bereshchenko, O. R., Gu, W. \& Dalla-Favera, R. Acetylation inactivates the transcriptional repressor BCL6. Nature Genet. 32, 606-613 (2002).

19. Grossman, S. R. et al. Polyubiquitination of $\mathrm{p} 53$ by a ubiquitin ligase activity of p300. Science 300, 342-344 (2003)

20. Shi, D. et al. CBP and p300 are cytoplasmic E4 polyubiquitin ligases for p53. Proc. Natl Acad. Sci. USA 106, 16275-16280 (2009).

21. Oike, Y. etal. Mice homozygous for a truncated form of CREB-binding protein exhibit defects in hematopoiesis and vasculo-angiogenesis. Blood 93, 2771-2779 (1999).

22. Yao, T. P. et al. Gene dosage-dependent embryonic development and proliferation defects in mice lacking the transcriptional integrator p300. Cell 93, 361-372 (1998).

23. Roelfsema, J. H. \& Peters, D. J. Rubinstein-Taybi syndrome: clinical and molecular overview. Expert Rev. Mol. Med. 9, 1-16 (2007).

24. Petrif, F. et al. Rubinstein-Taybi syndrome caused by mutations in the transcriptional co-activator CBP. Nature 376, 348-351 (1995).

25. Miller, R. W. \& Rubinstein, J. H. Tumors in Rubinstein-Taybi syndrome. Am. J. Med Genet. 56, 112-115 (1995)

26. Iyer, N. G., Ozdag, H. \& Caldas, C. p300/CBP and cancer. Oncogene 23, 4225-4231 (2004).

27. Gayther, S. A. et al. Mutations truncating the EP300 acetylase in human cancers. Nature Genet. 24, 300-303 (2000).

28. Ward, R., Johnson, M., Shridhar, V., van Deursen, J. \& Couch, F. J. CBP truncating mutations in ovarian cancer. J. Med. Genet. 42, 514-518 (2005)

29. Garbati, M. R., Alco, G. \& Gilmore, T. D. Histone acetyltransferase p300 is a coactivator for transcription factor REL and is C-terminally truncated in the human diffuse large B-cell lymphoma cell line RC-K8. Cancer Lett. 291, 237-245 (2010).

30. Shigeno, K. etal. Disease-related potential of mutations in transcriptional cofactors CREB-binding protein and p300 in leukemias. Cancer Lett. 213, 11-20 (2004).

31. Borrow, J. et al. The translocation $t(8 ; 16)(\mathrm{p} 11 ; \mathrm{p} 13)$ of acute myeloid leukaemia fuses a putative acetyltransferase to the CREB-binding protein. Nature Genet. 14, 33-41 (1996)

32. Rowley, J. D. etal. All patients with the $\mathrm{T}(11 ; 16)(q 23 ; \mathrm{p} 13.3)$ that involves MLL and CBP have treatment-related hematologic disorders. Blood 90, 535-541 (1997).

33. Sobulo, O. M. et al. MLL is fused to CBP, a histone acetyltransferase, in therapyrelated acute myeloid leukemia with a t(11;16)(q23;p13.3). Proc. Natl Acad. Sci. USA 94, 8732-8737 (1997)

34. Mullighan, C. G. et al. CREBBP mutations in relapsed acute lymphoblastic leukaemia. Nature doi: 10.1038/nature09727 (this issue).

35. Liu, X. et al. The structural basis of protein acetylation by the p300/CBP transcriptional coactivator. Nature 451, 846-850 (2008).

36. Phan, R. T. \& Dalla-Favera, R. The BCL6 proto-oncogene suppresses $p 53$ expression in germinal-centre B cells. Nature 432, 635-639 (2004).
37. Tang, Y., Zhao, W., Chen, Y., Zhao, Y. \& Gu, W. Acetylation is indispensable for p53 activation. Cell 133, 612-626 (2008).

38. Kwok, R. P. et al. Nuclear protein CBP is a coactivator for the transcription factor CREB. Nature 370, 223-226 (1994).

39. Kasper, L. H. etal. CBP/p300 double null cells reveal effect of coactivator level and diversity on CREB transactivation. EMBO J. 29, 3660-3672 (2010).

40. Bordoli, L. et al. Functional analysis of the p300 acetyltransferase domain: the PHD finger of $\mathrm{p} 300$ but not of CBP is dispensable for enzymatic activity. Nucleic Acids Res. 29, 4462-4471 (2001).

41. Xu, W. etal. Global transcriptional coactivators CREB-binding protein and $\mathrm{p} 300$ are highly essential collectively but not individually in peripheral B cells. Blood 107, 4407-4416 (2006)

42. Kung, A. L. et al. Gene dose-dependent control of hematopoiesis and hematologic tumor suppression by CBP. Genes Dev. 14, 272-277 (2000).

43. Legube, G. \& Trouche, D. Regulating histone acetyltransferases and deacetylases. EMBO Rep. 4, 944-947 (2003).

44. Phan, R. T., Saito, M., Basso, K., Niu, H. \& Dalla-Favera, R. BCL6 interacts with the transcription factor Miz-1 to suppress the cyclin-dependent kinase inhibitor p21 and cell cycle arrest in germinal center B cells. Nature Immunol. 6, 1054-1060 (2005).

45. Stimson, L., Wood, V., Khan, O., Fotheringham, S. \& La Thangue, N. B. HDAC inhibitor-based therapies and haematological malignancy. Ann. Oncol. 20, 1293-1302 (2009)

Supplementary Information is linked to the online version of the paper at www.nature.com/nature.

Acknowledgements We thank Q. Shen and the Molecular Pathology Shared Resource of the Herbert Irving Comprehensive Cancer Center at Columbia University for histology service; W. Gu for discussions and reagents; Y. Tang, M. Li and D. Chao for suggestions; V. Bardwell for the Bcl6 reporter construct; and R. S. K. Chaganti for sharing unpublished information. Whole-exome capture and sequencing were conducted at Roche NimbleGen and 454 Life Sciences. Automated DNA sequencing was performed at Genewiz Inc. This work was supported by NIH grants PO1-CA092625 and R01-CA37295 (to R.D.-F.), a Specialized Center of Research grant from the Leukemia and Lymphoma Society (to R.D.-F.), NIH grant DE018183, a Cancer Center (CORE) support grant P30 CA021765, and the American Lebanese Syrian Associated Charities of St Jude Children's Research Hospital (to P.K.B.), the Northeast Biodefence Center (U54-Al057158) and the National Library of Medicine (1R01LM010140-01) (to R.R.), and the AIRC Special Program Molecular Clinical Oncology - 5 per mille (contract number 10007, Milan) (to G.G.). A. Chiarenza is on leave from the Division of Hematology, Ospedale Ferrarotto, University of Catania, Catania, Italy. L.P. is on leave from the University of Perugia Medical School, Perugia, Italy.

Author Contributions L.P. and R.D.-F. designed the study and wrote the manuscript, with contributions from all authors. L.P. designed and conducted experiments, analysed data and coordinated the study. D.D.-S. designed and conducted experiments, and analysed immunohistochemistry data. A. Chiarenza, G.F. and A.G. conducted CREBBP/EP300 amplification and sequencing analysis. L.H.K., S.L. and P.K.B. were responsible for the experiments in MEF cells. H.T. performed immunohistochemistry and immunofluorescence staining of human tissue biopsies. V.V.M. developed FISH assays and analysed cytogenetic data. C.G.M. and J.M. analysed microarray data. A. Chadburn, D.R. and G.G. provided well-characterized patient samples. V.T. and R.R. developed algorithms and analysed high-throughput sequencing data.

Author Information The Affymetrix expression data reported in this paper have been deposited in the NCBI Gene Expression Omnibus (GEO) database (Series Accession Number GSE12195). The SNP Array 6.0 data and the whole exome sequencing data from the seven DLBCL cases have been deposited in dbGaP under accession number phs000328.v1.p1. Reprints and permissions information is available at www.nature.com/reprints. The authors declare no competing financial interests. Readers are welcome to comment on the online version of this article at www.nature.com/nature. Correspondence and requests for materials should be addressed to L.P. (Ip171@columbia.edu) or R.D.-F. (rd10@columbia.edu). 


\section{METHODS}

DNA extraction, amplification and sequencing. Genomic DNA was extracted by standard methods, and whole-genome amplification was performed using the RepliG kit (Qiagen) according to the manufacturer's instructions. Sequences for all annotated exons and flanking introns of CREBBP and EP300 were obtained from the UCSC Human Genome database, using the corresponding mRNA accession number as a reference (NM_004380.2 and NM_001429.3, respectively). PCR primers, located $\geq 50$ bp upstream or downstream to target exon boundaries, were designed in the Primer 3 program (http://frodo.wi.mit.edu/primer3/) and filtered using UCSC In Silico PCR (http://genome.ucsc.edu) to exclude pairs yielding more than a single product (Supplementary Tables 5 and 6). Purified amplicons were sequenced directly from both strands as described ${ }^{2}$, and compared to the corresponding germline sequences, using the Mutation Surveyor Version 2.41 software package (Softgenetics; http://www.softgenetics.

com). Somatic mutations were confirmed on independent PCR products obtained from high-molecular-weight genomic DNA. Synonymous mutations, previously reported polymorphisms (Human dbSNP Database at NCBI, Build 130, and Ensembl Database) and changes present in the matched normal DNA, when available, were excluded. In cases carrying multiple events within a single gene, the allelic distribution of the mutations was determined by cloning and sequencing PCR products obtained from cDNA and spanning both events $(n=10$ clones each).

Northern blot analysis of CREBBP and EP300 expression. Total RNA (12 $\mu \mathrm{g})$ was extracted from exponentially growing cell lines by TRIzol (Invitrogen) and Northern blot analysis was performed according to standard procedures, with radiolabelled probes corresponding to a $0.9-\mathrm{kb}$ fragment of the human $C R E B B P$ cDNA (region 204-1143, GenBank accession number NM_004380.2) or a 1.2-kb fragment of the human EP300 cDNA (region 6277-7524, GenBank accession number NM_001429.3), and GAPDH as a control for loading. CREBBP and EP300 expression levels were then quantitated by Phosphorimager analysis and normalized with the GAPDH levels.

Tissue microarrays, immunohistochemistry and immunofluorescence analysis. DLBCL tissue microarrays were constructed according to standard procedures and analysed by immunohistochemistry, using rabbit polyclonal antibodies directed against the N-terminus of CREBBP (A22) or EP300 (N15) (Santa Cruz Biotechnology). Cases were scored as positive if $\geq 20 \%$ tumour cells were stained by the antibody. Immunofluorescence analysis of CREBBP expression in conditional Crebbp/Ep300 double-knockout MEFs was performed three days after deletion of the endogenous loci and five days after infection with HA-tagged CREBBP retrovirus, using the CREBBP antibody (A22). The specificity of both the A22 and the N15 antibodies had been previously validated on paraffinembedded cell pellets from HEK293T cells transfected with control and CREBBP-specific short hairpin RNAs as well as by immunofluorescence staining of Crebbp/Ep300 double-knockout MEFs (data not shown).

Protein extraction and western blot analysis. Whole-cell extracts were prepared in RIPA buffer containing protease inhibitors as described ${ }^{18}$ and were analysed by western blotting according to standard methods, using the following primary antibodies: anti-FLAG/M2 (Sigma), anti-HA (3F10) (Roche), anti-BCL6 (GI191E/ A8, Cell Marque), rabbit anti-acetyl lysines (Cell Signaling), anti-acetylated p53 (kind gift of W. Gu), anti-p53 (DO1), anti-CREBBP (A22), anti-EP300 (N15) (all from Santa Cruz Biotechnology), anti-GFP (JL-8) (Clontech), anti- $\beta$-actin (clone $\mathrm{AC}-15)$ and anti- $\beta$-tubulin (clone B-5-1-2) (both from Sigma). Proteins were resolved by SDS-polyacrylamide gel electrophoresis (SDS-PAGE) in 3-8\% NuPAGE Tris-Acetate gels (Invitrogen), and visualized using a chemiluminescence detection kit (Pierce) as recommended by the manufacturer.

Transient transfections/reporter gene assays. Transient transfections were performed in HEK293T cells using polyethylenimine, as described ${ }^{46}$. For reporter assays, cells were seeded on a 24 -well plate and transfected using $100 \mathrm{ng}$ of a luciferase reporter construct driven by a PGL3-SV40-based backbone with five BCL6 binding sites ${ }^{47}, 2.5 \mathrm{ng}$ of pRL-SV40 (Promega), $1 \mathrm{ng}$ of BCL6-encoding plasmid and the indicated doses of wild-type and mutant CREBBP expression vectors. The total amount of transfected DNA was kept constant in each experiment by adding pCMV-HA or pcDNA3 vector sequences to a final amount of $600 \mathrm{ng}$ per well, and $3.5 \mu \mathrm{g}$ of polyethylenimine. All experiments were performed in duplicate and luciferase activities were measured $36 \mathrm{~h}$ after transfection using the Dual-Luciferase Reporter Assay System (Promega), according to the manufacturer's instructions. Given the known effects of CREBBP on the SV40 promoter and on other regulatory sequences, the response of the BCL6 reporter was normalized first to that of a SV40-Renilla reporter construct, and then to the basal activity of CREBBP on the 5X-BCL6 reporter, in the absence of BCL6. Data are expressed as differences relative to the basal activity of the reporter construct (set to 1) after the above-mentioned normalization.

Co-immunoprecipitation assays. To assess the interaction between CREBBP and BCL6 or p53, HEK293T cells were co-transfected with plasmids encoding for the two proteins, together with wild-type or mutant CREBBP-HA. Thirty-six hours after transfection (or twenty-four hours for p53), cells were lysed in immunoprecipitation buffer (50 mM Tris, pH 7.0, $250 \mathrm{mM} \mathrm{NaCl}, 1 \mathrm{mM}$ EDTA, $1 \%$ Triton X-100, $0.05 \%$ NP40, $10 \mathrm{mM}$ sodium fluoride, $0.1 \mathrm{mM}$ sodium orthovanadate and protease inhibitor cocktail) (from Sigma), and the cleared lysates were incubated overnight at $4{ }^{\circ} \mathrm{C}$ with anti-HA or anti-Flag/M2 beads (Sigma). Immunocomplexes were eluted by incubating the beads in immunoprecipitation buffer containing HA or 3X-Flag peptide, respectively (Sigma). A fraction of the final eluates was resolved by SDS-PAGE and analysed by western blot.

In vitro acetyl-transferase assays. Recombinant GST-p53 and CREBBP-HA proteins were obtained as described in detail in the Supplementary Information and used for in vitro acteylation assays according to published protocols, with minor modifications ${ }^{14,48,49}$. Briefly, $10-24 \mathrm{ng}$ of recombinant CREBBP-HA and $100 \mathrm{ng}$ of GST-p53 were combined in $40 \mu \mathrm{l}$ reactions containing $50 \mathrm{mM}$ Tris- $\mathrm{Cl}$ (pH 8.0), 10\% glycerol, 1 mM DTT, 1 mM PMSF, 0.1 mM EDTA, $10 \mathrm{mM}$ butyric acid (Sigma), and variable amounts of acetyl-CoA (Sigma) ( $2 \mathrm{mM}$ to $25 \mathrm{nM})$. As shown in Supplementary Fig. 6, acetyl-CoA concentrations as low as $25 \mathrm{nM}$ were sufficient to obtain efficient acetylation of the substrate by CREBBP. Reactions were performed for $60 \mathrm{~min}$ at $30^{\circ} \mathrm{C}$, and stopped by addition of an equal volume of $2 \times$ Laemmli buffer followed by heating for $10 \mathrm{~min}$ at $70^{\circ} \mathrm{C}$. A fraction of the final product was resolved by SDS-PAGE in 3-8\% Tris-Acetate gradient gels (Invitrogen) and analysed by western blotting.

Reconstitution and analysis of conditional Crebbp/Ep300 double-knockout MEFs. The protocols for MEF isolation, cell culture and retroviral transduction, as well as the mouse Crebbp (CBP)-HA retroviral construct used as the template for the generation of various CREBBP mutants have been described previously ${ }^{50}$. In this system, Cre-mediated recombination induces expression of YFP, allowing for specific identification of the deleted cells. Crebbp ${ }^{\text {flox } / f l o x} ; E p 300^{\text {flox } / f l o x} ; Y F P^{+}$ MEFs were first infected with retroviruses encoding either wild-type or selected CREBBP point mutants, and the endogenous Crebbp flox and Ep $300^{\text {flox }}$ loci were deleted after $48 \mathrm{~h}$ by infection with Cre-expressing adenovirus (Ad-Cre). The $\mathrm{W} 1502 \mathrm{~A} / \mathrm{Y} 1503$ S HAT-dead mutation was used as negative control ${ }^{40}$. In all experiments, transduction efficiencies were $70 \%$ or higher, as assessed by immunofluorescence analysis of HA-positive cells three days after deletion of endogenous Crebbp/Ep300 and five days after retroviral infection. To examine cAMPdependent gene expression, MEFs were cultured for $16 \mathrm{~h}$ in DMEM containing $0.1 \%$ FBS and treated for 90 min with $10 \mu \mathrm{M}$ forskolin $+100 \mu \mathrm{M}$ IBMX (or ethanol vehicle) before harvesting in TRIzol (Invitrogen); quantitative RT-PCR assays were performed as reported ${ }^{50}$. Expression of Crebbp and Ep300 was verified by immunofluorescence analysis using rabbit polyclonal anti-CREBBP (A22) and anti-EP300 (N20) antibodies (Santa Cruz Biotechnology). Analysis of H3K18 acetylation was performed as described, using the anti-H3K18Ac antibody (ab1191) (Abcam) and the HA-11 monoclonal antibody against the HA epitope (Boehringer Mannheim). Confocal images were taken using the same settings for all mutants and mean intensity ratios for individual nuclei were collected using SlideBook 5 software. Nuclei in which the Crebbp-HA signal was at least 2.5 -fold above background were used to calculate the ratio of the H3K18Ac mean signal intensity to the Crebbp-HA mean signal intensity. For the growth assays shown in Supplementary Fig. 11, Crebbp $p^{\text {flox/flox }} ; E p 300^{\text {flox/flox }} ; Y_{F P} P^{+}$MEFs were infected with CREBBP retroviruses $48 \mathrm{~h}$ before Ad-Cre-mediated deletion of endogenous Crebbp and Ep300. From each reconstituted population, equivalent numbers of $\mathrm{YFP}^{+}$(double-knockout) MEFs were seeded at day 1 (that is, the day following overnight Ad-Cre treatment), and the total number of $\mathrm{YFP}^{+}$cells was calculated on day 11 from the total cell number, based on the percentage of $\mathrm{YFP}^{+}$cells as assessed by flow cytometric analysis.

46. Bieber, T. \& Elsasser, H. P. Preparation of a low molecular weight polyethylenimine for efficient cell transfection. Biotechniques 30, 74-77,-80-81 (2001).

47. Huynh, K. D., Fischle, W., Verdin, E. \& Bardwell, V. J. BCoR, a novel corepressor involved in BCL-6 repression. Genes Dev. 14, 1810-1823 (2000).

48. Kuninger, D., Lundblad, J., Semirale, A. \& Rotwein, P. A non-isotopic in vitro assay for histone acetylation. J. Biotechnol. 131, 253-260 (2007).

49. Tang, Y., Luo, J., Zhang, W. \& Gu, W. Tip60-dependent acetylation of p53 modulates the decision between cell-cycle arrest and apoptosis. Mol. Cell 24, 827-839 (2006).

50. Bedford, D. C., Kasper, L. H., Fukuyama, T. \& Brindle, P. K. Target gene context influences the transcriptional requirement for the KAT3 family of CBP and p300 histone acetyltransferases. Epigenetics 5, 9-15 (2010). 